\title{
On likely values of the cosmological constant
}

\author{
Jaume Garriga \\ IFAE, Departament de Fisica, Universitat Autonoma de Barcelona, 08193 Bellaterra (Barcelona), Spain \\ and Institute of Cosmology, Department of Physics and Astronomy, Tufts University, Medford, Massachusetts 02155
}

Alexander Vilenkin

Institute of Cosmology, Department of Physics and Astronomy, Tufts University, Medford, Massachusetts 02155

(Received 10 September 1999; published 13 March 2000)

\begin{abstract}
We discuss models in which the smallness of the effective vacuum energy density $\rho_{\Lambda}$ and the coincidence of the time of its dominance $t_{\Lambda}$ with the epoch of galaxy formation $t_{G}$ are due to anthropic selection effects. In such models, the probability distribution for $\rho_{\Lambda}$ is a product of an a priori distribution $\mathcal{P}_{*}\left(\rho_{\Lambda}\right)$ and of the number density of galaxies at a given $\rho_{\Lambda}$ (which is proportional to the number of observers who will detect that value of $\rho_{\Lambda}$ ). To determine $\mathcal{P}_{*}$, we consider inflationary models in which the role of the vacuum energy is played by a slowly varying potential of some scalar field. We show that the resulting distribution depends on the shape of the potential and generally has a non-trivial dependence on $\rho_{\Lambda}$, even in the narrow anthropically allowed range. This is contrary to Weinberg's earlier conjecture that the a priori distribution should be nearly flat in the range of interest. We calculate the (final) probability distributions for $\rho_{\Lambda}$ and for $t_{G} / t_{\Lambda}$ in simple models with power-law potentials. For some of these models, the agreement with the observationally suggested values of $\rho_{\Lambda}$ is better than with a flat a priori distribution. We also discuss a quantum-cosmological approach in which $\rho_{\Lambda}$ takes different values in different disconnected universes and argue that Weinberg's conjecture is not valid in this case as well. Finally, we extend our analysis to models of quintessence, with similar conclusions.
\end{abstract}

PACS number(s): $98.80 . \mathrm{Cq}$

\section{INTRODUCTION}

The cosmological constant $\Lambda$ presents us with a number of perplexing problems (see [1] for a recent review). Particle physics models suggest that the natural value for $\Lambda$ is set by the Planck scale, $m_{p l} \sim 10^{19} \mathrm{GeV}$. The corresponding vacuum energy density is

$$
\rho_{\Lambda} \sim m_{p l}^{4}
$$

which is some 120 orders of magnitude greater than the observational bounds. This is what is usually called "the cosmological constant problem.' The discrepancy between the expected and observed values is so large that until recently it was almost universally believed that the cosmological constant must vanish. However, no convincing mechanism has yet been found that would set $\Lambda$ to zero.

It came as a total surprise when recent observations [2] provided strong evidence that the universe is accelerating, rather than decelerating, suggesting a non-zero cosmological constant. While there was still hope to explain a vanishing $\Lambda$, a small non-zero value appeared totally incomprehensible.

The observationally suggested values of $\Lambda$ correspond to $\rho_{\Lambda} \sim \rho_{M 0}$, where $\rho_{M 0}$ is the present density of matter. This brings yet another puzzle. It is difficult to understand why we happen to live at the epoch when $\rho_{M} \sim \rho_{\Lambda}$. That is why

$$
t_{0} \sim t_{\Lambda}
$$

where $t_{0}$ is the present time and $t_{\Lambda}$ is the time when the cosmological constant starts to dominate. Observers living at $t \ll t_{\Lambda}$ would find $\rho_{M} \gg \rho_{\Lambda}$, while observers living at $t \gg t_{\Lambda}$ would find $\rho_{M} \ll \rho_{\Lambda}$.

The only explanation of these puzzles that we are aware of attributes them to anthropic selection effects. In this approach, the cosmological constant is assumed to be a free parameter that can take different values in defferent parts of the universe, or perhaps in different disconnected universes. Weinberg [3] was the first to point out that not all values of $\Lambda$ are consistent with the existence of conscious observers [4]. In a spatially flat universe with a cosmological constant, gravitational clustering effectively stops at $t \sim t_{\Lambda}$, corresponding to the redshift $\left(1+z_{\Lambda}\right) \sim\left(\rho_{\Lambda} / \rho_{M 0}\right)^{1 / 3}$. At later times, the vacuum energy dominates and the universe enters a de Sitter stage of exponential expansion. An anthropic bound on $\rho_{\Lambda}$ can be obtained by requiring that it does not dominate before the redshift $z_{\max }$ when the earliest galaxies are formed:

$$
\rho_{\Lambda} \lesssim\left(1+z_{\max }\right)^{3} \rho_{M 0}
$$

Weinberg took $z_{\max } \sim 4$, which gives

$$
\rho_{\Lambda} \lesssim 100 \rho_{M 0}
$$

This is a dramatic improvement over Eq. (1), but it still falls short of the observational bound by a factor of about 30 .

The anthropic bound (4) specifies the value of $\rho_{\Lambda}$ which makes galaxy formation barely possible. However, as it was pointed out in $[5,6]$, the observers are where the galaxies are, and thus most of the observers will detect not these marginal 
values, but rather the values that maximize the number of galaxies. More precisely, the probability distribution for $\rho_{\Lambda}$ can be written as [5]

$$
d \mathcal{P}\left(\rho_{\Lambda}\right)=\mathcal{P}_{*}\left(\rho_{\Lambda}\right) \nu\left(\rho_{\Lambda}\right) d \rho_{\Lambda}
$$

Here, $\mathcal{P}_{*}\left(\rho_{\Lambda}\right) d \rho_{\Lambda}$ is the a priori distribution, which is proportional to the volume of those parts of the universe where $\rho_{\Lambda}$ takes values in the interval $d \rho_{\Lambda}$, and $\nu\left(\rho_{\Lambda}\right)$ is the average number of galaxies that form per unit volume with a given value of $\rho_{\Lambda}$. The calculation of $\nu\left(\rho_{\Lambda}\right)$ is a standard astrophysical problem; it can be done, for example, using the Press-Schechter formalism [7]. The a priori distribution $\mathcal{P}_{*}\left(\rho_{\Lambda}\right)$ should be determined from the theory of initial conditions, e.g., from an inflationary model or from quantum cosmology.

Martel, Shapiro and Weinberg [8] (see also [9]) presented a detailed calculation of $d \mathcal{P}\left(\rho_{\Lambda}\right)$. They first noted that $\mathcal{P}_{*}\left(\rho_{\Lambda}\right)$ can be expected to vary on some characteristic particle physics scale, $\Delta \rho_{\Lambda} \sim \eta^{4}$. The energy scale $\eta$ could be anywhere between the Planck scale and the electroweak scale, $\eta_{E W} \sim 10^{2} \mathrm{GeV}$. For any reasonable choice of $\eta, \Delta \rho_{\Lambda}$ exceeds the anthropically allowed range (4) by many orders of magnitude. Also, in the absence of a mechanism that sets the cosmological constant to zero, we may not expect any pronounced features in the probability distribution at low values of $\rho_{\Lambda}$. This suggests that we can set

$$
\mathcal{P}_{*}\left(\rho_{\Lambda}\right)=\text { const }
$$

in the range of interest. This argument is originally due to Weinberg [3], and we shall refer to Eq. (6) as Weinberg's conjecture. Once it is accepted, the problem reduces to the calculation of $\nu\left(\rho_{\Lambda}\right)$. Martel et.al. found that the resulting probability distribution is peaked at somewhat larger values of $\rho_{\Lambda}$ than observationally suggested. For the probability of $\rho_{\Lambda}$ being smaller or equal than the values indicated by the supernova data, it gives $\sim 5-10 \%$. In absolute terms, this is not a very large probability. However, the mere fact that it is non-negligible is rather impressive, in view of the large discrepancy in orders of magnitude between the a priori expected range for $\rho_{\Lambda}$ and its measured value.

Going back to the issue of the cosmic time coincidence, Eq. (2), this can also be explained by anthropic selection effects. Here is a sketch of the argument [10-13]. One first notes that the present time $t_{0}$ is bounded by

$$
t_{G} \lesssim t_{0} \lesssim t_{G}+t_{\star},
$$

where $t_{G}$ is the time of galaxy formation (which is also the time when most of the stars are formed) and $t_{\star}$ is the characteristic lifetime of habitable stars, $t_{\star} \sim 5-20$ Gyr. Observationally, giant galaxies were assembled at $z \sim 1-3$, or $t_{G}$ $\sim t_{0} / 3-t_{0} / 8$, that is, within an order of magnitude of $t_{0}$. Since $t_{G} \sim t_{\star}$, it follows from Eq. (7) that most observers live at the epoch when $t \sim t_{G}$, and the problem of explaining the coincidence $t_{0} \sim t_{\Lambda}$ is reduced to explaining why

$$
t_{G} \sim t_{\Lambda}
$$

The latter coincidence is not difficult to understand if we note that regions of the universe where $t_{\Lambda} \ll t_{G}$ do not form any galaxies at all.

The "coincidence" (8) can be expressed quantitatively by calculating the probability distribution for $t_{G} / t_{\Lambda}$. With a flat a priori distribution (6), one finds [12] that it has a broad peak in the range $0.3 \lesssim t_{G} / t_{\Lambda} \lesssim 5$ with a median at $t_{G} / t_{\Lambda}$ $\approx 1.5$. Thus, most observers will find themselves living in galaxies formed at $t_{G} \sim t_{\Lambda}$.

The probability distributions for $\rho_{\Lambda}$ and $t_{G} / t_{\Lambda}$ were calculated in Refs. $[8,12]$ by using Weinberg's conjecture. That is, without recourse to any particular model that would allow $\Lambda$ to vary and simply assuming the flat distribution (6). This is the beauty of the conjecture: if true, it would make the results independent of one's (necessarily speculative) assumptions about the very early universe, and therefore it would make the theory more predictive. It is important, however, to consider specific models with a variable vacuum energy and to check whether or not the conjecture is actually valid. This is one of our goals in the present paper.

In the next section we discuss models in which the role of the cosmological constant is played by a very slowly varying potential $V(\phi)$ of some scalar field $\phi$. We find that, unfortunately, Weinberg's conjecture is not generally valid in such models, and that the a priori distribution $\mathcal{P}_{*}\left(\rho_{\phi}\right)$ can be expected to be a non-trivial function of $\rho_{\phi}$ in the range of interest [here, $\left.\rho_{\phi} \equiv V(\phi)\right]$. We give some examples of potentials which do and do not satisfy the conjecture.

In Sec. III, we use simple models with power-law potentials, $V(\phi) \propto \phi^{n}$, to study the effect of a non-trivial a priori distribution on the final probability distribution for $\rho_{\phi}$ and on the cosmic time coincidence. For some of these models, we find that the agreement with the observationally suggested values of $\rho_{\phi}$ is better than what one gets from the calculations based on the flat distribution (6).

In Sec. IV we discuss models in which $\Lambda$ does not change throughout the universe but may take a range of values in different disconnected universes. Once again, we argue that Weinberg's conjecture is not likely to be valid in this case.

In Sec. V we extend our approach to models of quintessence. Our conclusions are briefly summarized in Sec. VI.

\section{SLOWLY VARYING POTENTIALS}

Suppose that what we perceive as a cosmological constant is in fact a potential $V(\phi)$ of some field $\phi(x)$. Observations will not distinguish between $V(\phi)$ and a true cosmological constant, provided that the kinetic energy of $\phi$ is small compared to $V(\phi)$,

$$
\dot{\phi}^{2} / 2 \ll V(\phi) \text {. }
$$

The evolution of $\phi$ is then described by the slow roll equation

$$
3 H \dot{\phi} \approx-V^{\prime}(\phi)
$$

and Eq. (9) gives

$$
V^{\prime 2} \ll 18 H^{2} V .
$$


We want to require that this condition still applies at the time when $V(\phi)$ is about to dominate. Then

$$
H^{2} \sim 8 \pi V(\phi) / 3 m_{p l}^{2},
$$

and Eq. (11) yields

$$
\left|V^{\prime}(\phi)\right| \ll 12 V(\phi) / m_{p l} .
$$

The dynamics of the field $\phi$ during inflation are strongly influenced by quantum fluctuations, causing different regions of the universe to thermalize with different values of $\phi$. Spatial variation of $\phi$ is thus a natural outcome of inflation. If $V(\phi)$ is sufficiently small, its back reaction on the rate of inflationary expansion is negligible, and all values of $\phi$ are equally probable,

$$
d \mathcal{P}_{*}(\phi) \propto d \phi .
$$

The condition for negligible back reaction is [14]

$$
m_{p l}^{2} V^{\prime 2} H^{4} / V^{3} \gg 1,
$$

where here $H$ is the Hubble rate during inflation.

Let us now recall that Weinberg's conjecture was motivated by the fact that the anthropically allowed range of $\rho_{\phi}$ is very small compared to the natural range of variation of $\rho_{\phi}$. One could expect that in this small range $V(\phi)$ can be approximated by a linear function. With an appropriate choice for the origin of $\phi$,

$$
V(\phi)=\kappa \phi .
$$

Then Eq. (14) implies a flat distribution for the vacuum energy density $\rho_{\phi} \equiv V(\phi)$,

$$
d \mathcal{P}_{*}\left(\rho_{\phi}\right) \propto d \rho_{\phi} .
$$

However, in our case Eq. (13) applied to the present time requires that the slope of $V(\phi)$ should be extremely small. The present Hubble rate is $H_{0} \sim 10^{-61} m_{p l}$, so using Eqs. (13) and (12) we have $\kappa \lesssim 10^{-122} m_{p l}^{3}$. As a result, a small range of $V(\phi)$ may correspond to a very large range of $\phi$. Indeed, it follows from Eq. (13) that

$$
\Delta \phi \sim V / V^{\prime} \gg m_{p l} .
$$

The natural range of $\phi$ in particle physics models is $\Delta \phi$ $\leqslant m_{p l}$, and there seems to be no reason to expect the slope of $V(\phi)$ to remain constant over the super-Planckian range (18).

Thus, we conclude that (i) models with variable $\rho_{\phi}$ can be easily constructed in the framework of inflationary cosmology and that (ii) Weinberg's conjecture (6) will not generally apply in this class of models. In the general case, assuming negligible back reaction, Eq. (14) yields

$$
d \mathcal{P}_{*}\left(\rho_{\phi}\right) \propto\left[V^{\prime}(\phi)\right]^{-1} d \rho_{\phi} .
$$

We now discuss some examples of potentials that do and do not satisfy the conjecture.

\section{Some examples}

We first consider a scalar field with a quadratic potential,

$$
V(\phi)=\rho_{\Lambda}+\frac{\mu^{2}}{2} \phi^{2}
$$

where $\rho_{\Lambda}$ is a "true" cosmological constant, which is assumed to be large. We assume also that $\rho_{\Lambda}$ and $\mu^{2}$ have opposite signs, so that the two terms in Eq. (20) partially cancel one another in some parts of the universe.

The cancellation occurs for $\phi \sim \sqrt{\rho_{\Lambda}} / \mu$, and Eqs. (13), (12) give the condition $\mu \ll H_{0}^{2} m_{p} / \sqrt{\rho_{\Lambda}}$, where $H_{0}$ $\sim 10^{-61} m_{p l}$ is the present Hubble rate. With $\rho_{\Lambda} \sim m_{p}^{4}$, this gives

$$
|\mu| \ll 10^{-122} m_{p l} .
$$

Thus, an exceedingly small mass scale must be introduced. On the other hand, the condition (15) for negligible back reaction imposes

$$
|\mu| \gg H_{0}^{3} H^{-2} \sim 10^{-169} m_{p l},
$$

where we have used $H \sim 10^{-7} m_{p l}$, corresponding to a grand unified theory (GUT) scale of inflation.

A critical reader may wonder at this point if anything is going to be achieved by explaining a cosmological constant $\rho_{\Lambda} \sim 10^{-120} \mathrm{~m}_{p l}^{4}$ in terms of a scalar field with a small mass of order $|\mu| \ll 10^{-122} m_{p l}$. However, potentials with very small masses or couplings could be generated through instanton effects. Suppose that we have a field $\phi$ with a flat potential, $V(\phi)=$ const, and that the radiative corrections to $V(\phi)$ vanish to all orders of perturbation theory, due to some symmetry. (For example, $\phi$ could be a Goldstone boson.) Suppose further that the symmetry is violated by instanton effects. Then $\phi$ will acquire a mass of the order $\mu^{2} \sim e^{-S} m_{p l}^{2}$, where $S$ is the instanton action. In order to have $|\mu| \ll 10^{-122} m_{p l}$, one needs $S \gtrsim 560$, which is not unreasonable.

The critic may still be unsatisfied and ask why the same kind of argument cannot be applied directly to the cosmological constant. One could imagine that $\rho_{\Lambda}=0$ to all orders of perturbation theory, due to some approximate symmetry, and that a small $\rho_{\Lambda} \propto \exp (-S)$ is induced by instantons. The problem with this scenario is that it does not explain the cosmic time coincidence (2). The instanton action $S$ should be fine-tuned so that $\Lambda$ starts dominating at the present time. Models with $\rho_{\Lambda}$ replaced by $V(\phi)$ are therefore preferred.

The potential (20) can be rewritten as

$$
V(\phi) \equiv \rho_{\phi}=\kappa\left(\phi-\phi_{0}\right)+\frac{\mu^{2}}{2}\left(\phi-\phi_{0}\right)^{2},
$$

where $\phi_{0}^{2}=-2 \rho_{\Lambda} / \mu^{2}$ and $\kappa=\mu^{2} \phi_{0}$. Then, using Eq. (19) in the vicinity of $\phi=\phi_{0}$ we have

$$
d \mathcal{P}_{*}\left(\rho_{\phi}\right) \propto\left(1+2 \frac{\mu^{2}}{\kappa^{2}} \rho_{\phi}\right)^{-1 / 2} d \rho_{\phi}=\left[1+\mathcal{O}\left(\rho_{\phi} / \rho_{\Lambda}\right)\right] d \rho_{\phi} .
$$


Since $\rho_{\phi} / \rho_{\Lambda} \ll 1$ in the anthropically allowed range, we conclude that Weinberg's conjecture applies to very good approximation in this case. The reason is that the cancellation of the two terms in Eq. (20) occurs at a very large value of $\phi \gg m_{p l}$ and the characteristic range of variation of a powerlaw potential is $\Delta \phi \sim \phi$. For the same reason, potentials of the form

$$
V(\phi)=\rho_{\Lambda}+A \phi^{n}
$$

can also be expected to satisfy the conjecture.

To give an example of a potential for which Weinberg's conjecture is not satisfied, consider a "washboard" potential

$$
V(\phi)=\rho_{\Lambda}+A \phi+B \sin (\phi / M),
$$

where $M \lessgtr m_{p l}$ is some particle physics scale and the constants $A$ and $B$ are small enough to satisfy the condition (13). In this case, Eq. (19) gives a distribution

$$
\mathcal{P}_{*} \propto[A+(B / M) \cos (\phi / M)]^{-1},
$$

which is not flat, unless $B / A M \ll 1$.

\section{POWER-LAW POTENTIALS}

We shall now consider a different situation, where the true cosmological constant has been set equal to zero by some unspecified mechanism, but the potential energy of a scalar field (whose minimum is at $V=0$ ) induces a small effective cosmological constant. Since the minimum of the potential is at $\rho_{\phi}=0$, Weinberg's conjecture is not expected to apply in this case.

To illustrate the effects of a non-trivial a priori distribution $\mathcal{P}_{*}\left(\rho_{\phi}\right)$, we shall calculate the probability distributions for $\rho_{\phi}$ and $t_{G} / t_{\phi}$ in the simple case of a power-law potential,

$$
V(\phi) \propto \phi^{n} .
$$

Familiar examples of such potentials are

$$
V(\phi)=\frac{1}{2} m^{2} \phi^{2}
$$

and

$$
V(\phi)=\frac{1}{4} \lambda \phi^{4} .
$$

They can be suitable for our purposes only if the parameters $m$ and $\lambda$ are very small. Indeed, Eqs. (12) and (13) require $\phi \gg m_{p l} / 6, \quad m \ll 3 H_{0}$ for Eq. (29) and $\phi \gg m_{p l} / 3, \quad \lambda$ $\ll 40 H_{0}^{2} / m_{p l}^{2}$ for Eq. (30). Thus, we obtain the constraints $m \ll 10^{-61} m_{p l}$ and $\lambda \ll 10^{-119}$. The condition (15) for negligible back reaction will impose lower bounds on these parameters. For the quadratic potential it requires $m$ $\gg 10^{-108} m_{p l}$ and for the quartic it gives $\lambda \gg 10^{-310}$. Here, as in the previous section, we have used $H \approx 10^{-7} m_{p l}$, corresponding to a GUT-scale inflation, and $V \sim m_{p l}^{2} H_{0}^{2}$, with $H_{0} \approx 10^{-61} m_{p l}$. Inflation at a lower energy scale will impose somewhat tighter bounds. Again, the small masses and couplings can be induced by instanton effects.

In what follows we shall assume that back-reaction effects are negligible (Otherwise, $\mathcal{P}_{*}(\phi)$ can be calculated by solving the Fokker-Planck equation of stochastic inflation; see Ref. [15].) Then, substituting Eq. (28) in Eq. (19) we have

$$
d \mathcal{P}_{*}\left(\rho_{\phi}\right) \propto \rho_{\phi}^{-(n-1) / n} d \rho_{\phi} .
$$

For $n>1$, the probability density grows towards smaller values of $\rho_{\phi}$ and has an integrable singularity at $\rho_{\phi}=0$. For $n$ $=1$, the distribution is flat, as in Weinberg's conjecture (6). For $0<n<1$, it grows towards large values of $\rho_{\phi}$. Finally, for $n<0$ the distribution has a non-integrable singularity at $\rho_{\phi}=0$; in this case $\rho_{\phi}=0$ with a $100 \%$ probability. As we mentioned in the Introduction, for a flat a priori distribution $\mathcal{P}_{*}\left(\rho_{\phi}\right)=$ const $(n=1)$, the full probability distribution (5) is peaked at a somewhat larger value of $\rho_{\phi}$ than observationally indicated. The agreement with observations may be improved if $\mathcal{P}_{*}\left(\rho_{\phi}\right)$ grows towards smaller values as for $n$ $>1$. We shall therefore concentrate on this case.

Following [12], we introduce the variable

$$
x=\frac{\Omega_{\phi}}{\Omega_{M}}=\sinh ^{2}\left(\frac{t}{t_{\phi}}\right),
$$

where $\Omega_{M}$ and $\Omega_{\phi}$ are, respectively, the densities of matter and of the scalar potential in units of the critical density, and $t_{\phi}$ is the time of $\phi$-domination. For convenience, we have defined $t_{\phi}$ as the time at which $\Omega_{\phi}=\sinh ^{2}(1) \Omega_{M}$ $\approx 1.38 \Omega_{M}$. At the time of recombination, for values of $\rho_{\phi}$ within the anthropic range, $x_{\text {rec }} \approx \rho_{\phi} / \rho_{\text {rec }} \ll 1$, where the matter density at recombination, $\rho_{\text {rec }}$, is independent of $\phi$. We can therefore express the probability distribution for $\rho_{\phi}$ as a distribution for $x_{r e c}$,

$$
d \mathcal{P}\left(x_{r e c}\right) \propto \nu\left(x_{r e c}\right) x_{r e c}^{1 / n} d \ln x_{r e c},
$$

where $\nu\left(x_{\text {rec }}\right)$ is the number of galaxies formed per unit volume in regions with a given value of $x_{\text {rec }}$. For $n=1$ the calculation of the distribution (33) was discussed in detail by Martel et al. [8]. In [12] we gave a simplified version of their calculation, which we generalize here to the case $n>1$.

In a universe where the effective cosmological constant is non-vanishing, a primordial overdensity will eventually collapse provided that its value at the time of recombination exceeds a certain critical value $\delta_{c}^{r e c}$. In the spherical collapse model this is estimated as $\delta_{c}^{r e c}=1.13 x_{r e c}^{1 / 3}$ (see e.g., [16]). Hence, the fraction of matter that eventually clusters in galaxies can be roughly approximated as $[7,16]$

$$
\nu\left(x_{r e c}\right) \approx \operatorname{erfc}\left(\frac{\delta_{c}^{r e c}}{\sqrt{2} \sigma_{r e c}\left(M_{g}\right)}\right) \approx \operatorname{erfc}\left(\frac{.80 x_{r e c}^{1 / 3}}{\sigma_{r e c}\left(M_{g}\right)}\right) .
$$

Here, erfc is the complementary error function and $\sigma_{r e c}\left(M_{g}\right)$ is the dispersion in the density contrast at the time of recombination on the relevant galactic mass scale $M_{g} \sim 10^{12} M_{\odot}$. 


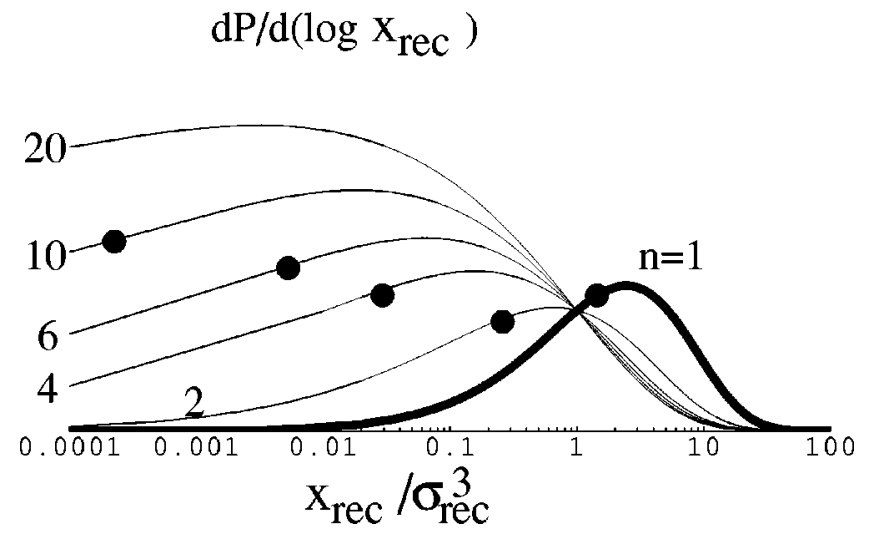

FIG. 1. The probability distribution (33) for the effective cosmological constant $\rho_{\phi}$, for different values of $n$. As explained in the text, an observed value of $\Omega_{\phi} \approx .7$ corresponds to $x_{\text {rec }} / \sigma_{\text {rec }}^{3}$ $\approx .1$. There is at present some uncertainty in this estimate, because a number of assumptions must be made in order to infer the value of $\sigma_{\text {rec }}$ from observations. Notice, however, that this value lies at the tail of the $n=1$ curve, corresponding to Weinberg's conjecture (a flat a priori distribution). On the other hand, for $2 \leqslant n \leqslant 5$ the value $x_{r e c} / \sigma_{r e c}^{3} \approx .1$ is well within the broad peak of the distribution. Thus, it is possible that a departure from Weinberg's conjecture may actually fit the observations better (more so if it turns out that the cosmological constant is smaller than .7). The median of each distribution is indicated by a round bead.

The logarithmic distribution $d \mathcal{P} / d \ln x_{\text {rec }}=x_{\text {rec }}^{1 / n} \nu\left(x_{\text {rec }}\right)$ is plotted in Fig. 1 for several values of $n$. For $n=1$ it has a rather broad peak which spans two orders of magnitude in $x_{\text {rec }}$, with a maximum at

$$
x_{\text {rec }}^{\text {peak }} \approx 2.45 \sigma_{\text {rec }}^{3} .
$$

As noted by Martel et al. [8], the parameter $\sigma_{r e c}$ can be inferred from observations of the cosmic microwave background anisotropies, although its value depends on the assumed value of the effective cosmological constant in our part of the universe today. For instance, assuming that the present cosmological constant is $\Omega_{\phi, 0}=.8$, and the relevant galactic co-moving scale is in the range $R=(1-2) \mathrm{Mpc}$, Martel et al. found $\sigma_{r e c} \approx(2.3-1.7) \times 10^{-3}$. In this estimate, they also assumed a scale invariant spectrum of density perturbations, a value of $70 \mathrm{~km} \mathrm{~s}^{-1} \mathrm{Mpc}^{-1}$ for the present Hubble rate, and they defined recombination to be at redshift $z_{\text {rec }} \approx 1000$ (this definition is conventional, since the probability distribution for the cosmological constant does not depend on the choice of reference time). Thus, taking into account that $x$ scales like $(1+z)^{-3}$ in Eq. (35), one finds that the peak of the distribution for the cosmological constant today is at $x_{0}^{\text {peak }} \approx 29.8-12$. The value corresponding to the assumed $\Omega_{\phi, 0}=.8$ is $x_{0}=4$, certainly within the broad peak of the distribution and not far from its maximum.

However, if we assume instead that the measured value is $\Omega_{\phi, 0}=.7$, which corresponds to $x_{0}=2.3$, the new inferred values for $\sigma_{\text {rec }} \approx(3.3-2.4) \times 10^{-3}$ correspond to the peak value $x_{0}^{\text {peak }} \approx(88-34)$. In this case, for $n=1$, the measured value would be at the outskirts of the broad peak, where the

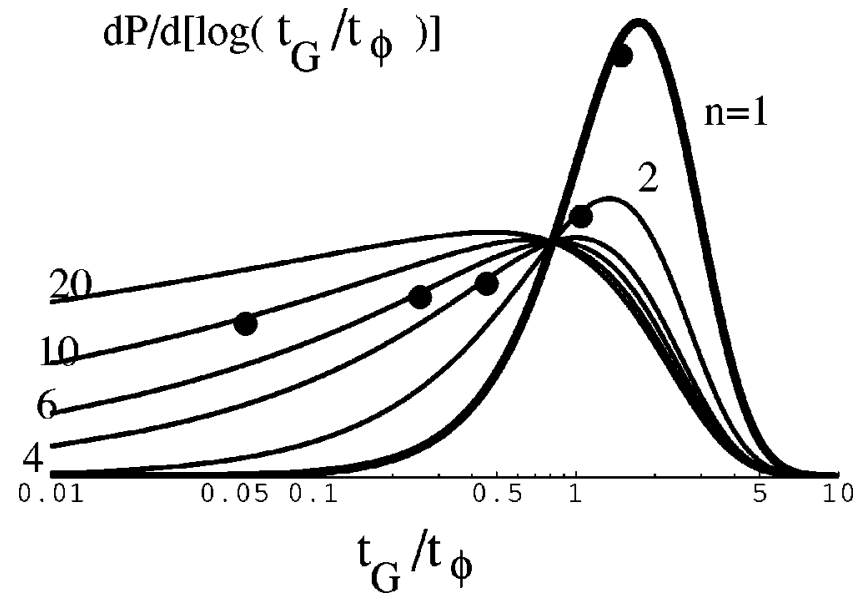

FIG. 2. Probability distribution for $t_{G} / t_{\phi}$, Eq. (36), for different values of $n$. The round beads indicate the median of each distribution. Note that the time coincidence $t_{G} \sim t_{\phi}$ is not unexpected for $1 \leqslant n \lesssim 10$.

logarithmic probability density is about an order of magnitude smaller than at the peak. Although this is still a significant probability, it is unfortunately somewhat low.

For a potential (28) with $n>1$, the peak in the distribution shifts to lower values of the effective cosmological constant, and therefore a measured value of $\Omega_{\phi, 0}=.7$ (which corresponds to $\left.x_{r e c} \sigma_{r e c}^{-3} \approx .1\right)$ becomes much better positioned. From Fig. 1, it is clear that for $2 \leqslant n \leqq 5$ this value lies well within the broad peak of the distribution. Thus we conclude that the violation of Weinberg's conjecture by a power-law potential with $n>1$ may actually lead to a better agreement with observations.

Let us now consider the issue of the time coincidence. Following our earlier computation [12] for the case $n=1$, we find that the probability distribution for $t_{G} / t_{\phi}$ is given by

$$
d \mathcal{P}\left(t_{G} / t_{\phi}\right) \propto[F(x)]^{3 / n-1} F^{\prime}(x) \frac{d x}{d \ln \left(t_{G} / t_{\phi}\right)} d \ln \left(t_{G} / t_{\phi}\right),
$$

where, here, $x=\sinh ^{2}\left(t_{G} / t_{\phi}\right)$ and

$$
F(x)=\frac{5}{6}\left(\frac{1+x}{x}\right)^{1 / 2} \int_{0}^{x} \frac{d \omega}{\omega^{1 / 6}(1+\omega)^{3 / 2}} .
$$

This distribution is shown in Fig. 2 for various values of $n$. For $n=1$ it has a broad peak which almost vanishes outside of the range $.1 \lesssim\left(t_{G} / t_{\phi}\right) \lesssim 10$. The maximum of the distribution is at $t_{G} / t_{\phi} \approx 1.7$ and the median value is at $t_{G} / t_{\phi} \approx 1.5$. Thus, most observers will find that their galaxies formed at $t \sim t_{\Lambda}$, which explains the time coincidence

$$
t_{G} \sim t_{\Lambda}
$$

As shown in Fig. 2, smaller values of $t_{G} / t_{\phi}$ become more likely as we increase $n$. However, values of $n \lesssim 10$ do not 
really spoil the coincidence (38), and even for $n$ as large as 30 , there is still a $5 \%$ probability for having $t_{G} / t_{\phi} \geqslant 1$.

\section{QUANTUM COSMOLOGY}

Let us now consider models with a true cosmological constant, $\rho_{\Lambda}=$ const, which takes the same value in the entire universe but may have different values in other disconnected universes. One example [19] is given by a four-index field $F_{\mu \nu \sigma \tau}$ whose value is undetermined by the field equations, $\partial_{\lambda} F_{\mu \nu \sigma \tau}=0$, and which gives a constant contribution to the vacuum energy,

$$
\rho_{\Lambda}=-(1 / 48) F_{\mu \nu \sigma \tau} F^{\mu \nu \sigma \tau}
$$

The a priori probability distribution for $\rho_{\Lambda}$ in this kind of models can be found in the framework of quantum cosmology [18]. One should calculate the cosmological wave function $\psi\left(\rho_{\Lambda}\right)$ which gives an amplitude for an inflationary universe to nucleate with a given value of $\rho_{\Lambda}$. In the semiclassical approximation,

$$
\psi \propto e^{ \pm S / 2}
$$

where $S$ is the action of the corresponding instanton. The upper sign in Eq. (40) is for the tunneling wave function and the lower sign for the Hartle-Hawking wave function. This choice of sign is a matter of some controversy [17], but it will not be important for our discussion here. The nucleation probability corresponding to Eq. (40) is

$$
\mathcal{P}_{\text {nucl }}\left(\rho_{\Lambda}\right) \propto \exp \left[ \pm S\left(\rho_{\Lambda}\right)\right]
$$

The instanton in Eq. (41) is a solution of Euclidean Einstein's equations (possibly with quadratic and higher-order curvature corrections) with a cosmological constant $\rho_{\Lambda}$ and a high-energy inflaton potential as a source. For small values of $\rho_{\Lambda}$, one can expect the instanton action to be independent of $\rho_{\Lambda}, \quad S\left(\rho_{\Lambda}\right) \approx$ const. We note, however, that different universes in the ensemble described by the wave function $\psi$ will generally have very different numbers of galaxies and, therefore, of observers. To take this into account, one has to use Eq. (5) with

$$
\mathcal{P}_{*}\left(\rho_{\Lambda}\right) \propto \mathcal{P}_{n u c l}\left(\rho_{\Lambda}\right) \mathcal{V}_{*}\left(\rho_{\Lambda}\right)
$$

where $\mathcal{V}_{*}\left(\rho_{\Lambda}\right)$ is the volume of the universe at the end of inflation, when the vacuum energy is thermalized. [The factor $\nu\left(\rho_{\Lambda}\right)$ in Eq. (5) should then be understood as the number of galaxies formed per unit thermalized volume.]

The right-hand side of Eq. (42) would be well defined if inflation had a finite duration, so that $\mathcal{V}_{*}\left(\rho_{\Lambda}\right)<\infty$. It is well known, however, that inflation is generically eternal [21,22]: at any time there are parts of the universe that are still inflating, and both inflating and thermalized volumes grow exponentially with time. In an ensemble of eternally inflating uni- verses, all volumes $\mathcal{V}_{*}$ become infinite in the limit $t \rightarrow \infty$, and Eq. (42) becomes meaningless. ${ }^{1}$

It appears reasonable, in this case, to look not at the total volume $\mathcal{V}_{*}$ but at the rate of its growth (which generally depends on $\rho_{\Lambda}$ ). With a cutoff at time $t$,

$$
\mathcal{V}_{*}\left(\rho_{\Lambda}, t\right) \propto \exp \left[\gamma\left(\rho_{\Lambda}\right) t\right]
$$

and the most probable value $\rho_{\Lambda}^{(*)}$ is found from $[5,24-29]$

$$
\gamma\left(\rho_{\Lambda}^{(*)}\right)=\max
$$

As time goes on, the volume of the universes with this preferred value of $\rho_{\Lambda}$ gets larger than the competition by an arbitrarily large factor, and thus in the limit $t \rightarrow \infty$ the probability for $\rho_{\Lambda}=\rho_{\Lambda}^{(*)}$ is equal to 1 ,

$$
\mathcal{P}_{*}\left(\rho_{\Lambda}\right) \propto \delta\left(\rho_{\Lambda}-\rho_{\Lambda}^{(*)}\right) .
$$

This is in a sharp contrast with Weinberg's conjecture (6).

There seems to be no reason to expect that the value of $\rho_{\Lambda}$ selected by the condition (44) will fall into the anthropic range. This approach is therefore unlikely to explain the smallness of $\rho_{\Lambda}$ or the cosmic time coincidence.

We also mention some alternative approaches. Hawking [19] suggested that the probability distribution for the observed values of $\rho_{\Lambda}$ is given by Eq. (41) with a minus sign in the exponential and with $S\left(\rho_{\Lambda}\right)=-3 / 8 \rho_{\Lambda}$, corresponding to a de Sitter instanton of energy density $\rho_{\Lambda}$,

$$
\mathcal{P} \propto \exp \left(3 / 8 \rho_{\Lambda}\right)
$$

Since the Lorentzian continuation of this instanton describes an empty universe dominated by the cosmological constant, it cannot be used to describe the nucleation of the universe, so Eq. (46) is hard to justify.

Coleman [20] suggested that the Euclidean path integral of quantum gravity is dominated by the lowest-energy de Sitter instantons connected by Planck-size wormholes. The resulting probability distribution is

$$
\mathcal{P} \propto \exp \left[\exp \left(3 / 8 \rho_{\Lambda}\right)\right]
$$

Both expressions (46),(47) have non-integrable peaks at $\rho_{\Lambda}$ $=0$ and thus do not satisfy Weinberg's conjecture.

\footnotetext{
${ }^{1}$ In fact this conclusion seems to apply even if the inflaton potential does not drive eternal inflation. After a finite period of inflation the cosmological constant will eventually dominate, driving a de Sitter-like phase. Recycling events [23] that create new regions of the inflating phase will then occur at a constant rate per unit spacetime volume, making the total thermalized volume an exponentially growing function of time.
} 


\section{QUINTESSENCE}

We finally comment on models of quintessence with "tracking", solutions which are now being extensively discussed in the literature [30]. These models require a scalar field $Q$ with a potential $V(Q)$ approaching zero at large values of $Q$. Note that this assumes that the cosmological constant problem has been solved by some mechanism, so that the true cosmological constant is set equal to zero (as in the case of power-law potentials discussed in Sec. III). A popular example of quintessence is an inverse power-law potential of the form

$$
V(Q)=\lambda M^{4+\beta} Q^{-\beta}
$$

with a constant $M \ll m_{p l}$. The quintessence field $Q$ approaches an attractor "tracking" solution and evolves towards larger values on a cosmological time scale $t$. When $Q$ becomes comparable to $m_{p l}$, the universe gets dominated by $V(Q)$, and the parameters of the model can be adjusted so that this happens at the present epoch.

It has been argued [31] that quintessence models do not suffer from the cosmic time coincidence problem, because the time $t_{Q}$ of $Q$-domination is not sensitive to the initial conditions. This time, however, does depend on the details of the potential $V(Q)$, and observers should be surprised to find themselves living at the epoch when quintessence is about to dominate. More satisfactory would be a model in which the potential depends on two fields, say $Q$ and $\phi$, with $\phi$ slowly varying in space, making the time of $Q$-domination positiondependent. We could choose, for example,

$$
V(Q, \phi)=\lambda M^{4+\beta-n} \phi^{n} Q^{-\beta}
$$

for $Q \gg M$ and $V(Q, \phi) \sim \lambda M^{4-n} \phi^{n}$ for $Q \lesssim M$.

For this model to work, the initial conditions for the fields $\phi$ and $Q$ at the end of inflation should be different: $\phi$ should be spread over a range $\Delta \phi \gg m_{p l}$ as before, while $Q$ should be concentrated at small values, $Q \ll m_{p l}$, so that it can get to the tracking solution. This can be arranged if $Q$ has a nonminimal coupling to the curvature, $\frac{1}{2} \xi R Q^{2}$. Then $Q$ acquires an effective mass $m_{Q}^{2}=12 \xi H^{2}$ during inflation, and its values immediately after inflation are concentrated in the range $Q^{2}$ $\lesssim H^{2} / \xi$ (bounds on on the time variation of the gravitational constant at late times require $\xi \lesssim 10^{-2}$ [32]). The field $\phi$ is assumed to be minimally coupled to the curvature $\left(\xi_{\phi}=0\right)$, and its values are randomized by quantum fluctuations during inflation. This results in a flat distribution (14), provided that $\lambda$ and $M$ are sufficiently small.

With these assumptions, a typical region of the universe after inflation will have $Q \ll m_{p l}$ and $\phi \gg m_{p l}$. In all such regions, $\phi$ will remain nearly constant, while $Q$ will evolve along the tracking solution, until the potential (49) dominates the universe. This happens at $Q \sim m_{p l}$. The energy density at the time of $Q$-domination is

$$
\rho_{Q} \sim \lambda M^{4+\beta-n} m_{p l}^{-\beta} \phi^{n} \propto \phi^{n}
$$

and the a priori distribution for $\rho_{Q}$ is

$$
d \mathcal{P}_{*}\left(\rho_{Q}\right) \propto \rho_{Q}^{-(n-1 / n)} d \rho_{Q}
$$

as in Eq. (31). The full distribution can be obtained as before using Eq. (5). Note, however, that the expression (34) for $\nu\left(\rho_{Q}\right)$ cannot be used in this case, because the evolution of density perturbations is different in models with an evolving $\rho_{Q}$ and with $\rho_{\Lambda}=$ const. Press-Schechter formalism has been applied to structure formation in quintessence models by Wang and Steinhardt [33], and their results can be easily adapted to the calculation of $\nu\left(\rho_{Q}\right)$ in a specific quintessence model. The cutoff of the growth of density perturbations at $t \sim t_{Q}$ in quintessence models is milder than that in models with a constant vacuum energy density, and we expect the peak of the probabilty distribution for $\rho_{Q}$ to be shifted dowards larger values. The qualitative character of the distribution is expected to be unchanged, and in particular the cosmic time coincidence (8) is likely to hold for a wide range of model parameters.

\section{CONCLUSIONS}

The results of our analysis include some bad news and some good news. The bad news is that Weinberg's conjecture (a flat a priori probability distribution $\mathcal{P}_{*}\left(\rho_{\Lambda}\right)$ ) is not generally valid. This conclusion applies both to models with slowly varying potentials and to models with an ensemble of disconnected universes having different (constant) values of $\rho_{\Lambda}$. We regard this as bad news because, without Weinberg's conjecture, the anthropic approach becomes less predictive.

In the quantum-cosmological approach, $\mathcal{P}_{*}\left(\rho_{\Lambda}\right)$ tends to select a single value of $\rho_{\Lambda}$. One can hope that this approach may provide an explanation for a vanishing true cosmological constant, but one would still have to find another mechanism to explain a small but nonzero effective cosmological constant. In the case of a slowly varying potential $V(\phi)$, the a priori distribution $\mathcal{P}_{*}$ depends on the shape of the potential, which is of course highly uncertain. (There is, however, a wide class of potentials for which the conjecture does apply.)

The good news is that the cosmic time coincidence (8) is not very sensitive to the shape of $V(\phi)$. For a power-law potential, $V(\phi) \propto \phi^{n}$, one finds that the probability distribution for $t_{G} / t_{\Lambda}$ is peaked at $t_{G} / t_{\Lambda} \sim 1$ in the wide range 1 $\lesssim n \lesssim 10$. Moreover, for values of $n$ in the range $2 \lesssim n \lesssim 5$, the peak of the probability distribution for $\rho_{\Lambda}$ is closer to the observationally suggested values than it is for $n=1$ (corresponding to the Weinberg's conjecture).

We have also suggested an extension of quintessence models in which the time of quintessence domination is determined by a slowly varying scalar field. The above conclusions apply to this class of models, with minor modifications.

A common objection to anthropic arguments is that they are not testable. It is therefore worth pointing out that models with a scalar field potential playing the role of the cosmological constant are falsifiable, at least in principle. Such models predict the existence of a nearly massless, minimally coupled scalar field. Fluctuations of this field are produced during inflation with the same spectrum as gravitons (and 
with half the energy density). Thus, for instance, if the energy density in gravity waves is found to be in the range marginally allowed by nucleosynthesis (as it may happen in some models of quintessential inflation [34]), the existence of a massless field would be ruled out; and with it the anthropic explanation for the time coincidence.

\section{ACKNOWLEDGMENTS}

We are grateful to Tom Banks, Pierre Binetruy and Steven Weinberg for useful discussions. This work was supported by CIRIT under grant 1998BEAI400244 (J.G.) and by the National Science Foundation (A.V.).
[1] V. Sahni and A. Starobinsky, "The Case for a Positive Cosmological Lambda-term," astro-ph/9904398.

[2] S. Perlmutter et al., Astrophys. J. 483, 565 (1997); S. Perlmutter et al., Bull. Am. Phys. Soc. 29, 1351 (1997); B. Schmidt et al., Astrophys. J. 507, 46 (1998); A. J. Riess et al., ibid. 116, 1009 (1998).

[3] S. Weinberg, Phys. Rev. Lett. 59, 2607 (1987).

[4] For an early attempt to apply anthropic arguments to the cosmological constant, see also P. C. W. Davis and S. Unwin, Proc. R. Soc. London A377, 147 (1981).

[5] A. Vilenkin, Phys. Rev. Lett. 74, 846 (1995).

[6] G. Efstathiou, Mon. Not. R. Astron. Soc. 274, L73 (1995).

[7] W. H. Press and P. Schechter, Astrophys. J. 187, 425 (1974).

[8] H. Martel, P. R. Shapiro, and S. Weinberg, Astrophys. J. 492, 29 (1998).

[9] S. Weinberg, in Critical Dialogues in Cosmology, edited by N. G. Turok (World Scientific, Singapore, 1997).

[10] A similar argument for the coincidence of the present time $t_{0}$ with the curvature domination time $t_{\Omega}$ in an open universe was given in [11].

[11] J. Garriga, T. Tanaka, and A. Vilenkin, Phys. Rev. D 60, 023501 (1999).

[12] J. Garriga, M. Livio, and A. Vilenkin, Phys. Rev. D 61, 023503 (2000).

[13] S. Bludman, "Vacuum Energy: If Not Now, Then When?', astro-ph/9907168.

[14] The expansion rate during inflation can be written as $H$ $+\delta H(\phi)$, where $\delta H(\phi) \approx 4 \pi V(\phi) / 3 m_{p l}^{2} H$. The $\phi$-dependent correction to $H$ will tend to distort the flat distribution, favoring larger values of $V(\phi)$. This effect becomes important on a time scale $\tau \sim 1 / \delta H$. On the other hand, quantum "diffusion", of $\phi$ will tend to keep the distribution flat. Quantum fluctuations of $\phi$ can be pictured as a random walk of step $\delta \phi \sim$ $\pm H$ per Hubble time $H^{-1}$, with steps taken independently in each horizon-size region $\left(\delta l \sim H^{-1}\right)$. The typical variation of $\phi$ due to this quantum random walk in a time interval $\tau$ is $\left(\Delta \phi_{q}\right)^{2} \sim H^{2} N \sim m_{p l}^{2} H^{4} / V(\phi)$, where $N \sim H \tau$ is the number of steps during the interval $\tau$. The back reaction can be neglected if $\Delta \phi_{q}$ is much greater than the relevant interval of $\phi, \Delta \phi$ $\sim V(\phi) / V^{\prime}(\phi)$. This gives

$$
\frac{V^{\prime 2} H^{4}}{V^{3}} \gg 1
$$

Consistency with Eq. (13) requires $V \ll H^{4}$, which is satisfied with a wide margin for anthropically allowed values of $V$ $\lesssim 100 \rho_{M 0}$ and all acceptable values of the inflationary expansion rate $H$. Hence, there is a wide range of slopes of the potential for which the back reaction effects are negligible.
However, for sufficiently small $V^{\prime}$, the above condition is violated, and the a priori probability $\mathcal{P}_{*}\left(\rho_{\phi}\right)$ should grow faster with $\rho_{\phi}$ than indicated by Eq. (19).

[15] V. Vanchurin, A. Vilenkin, and S. Winitzki, gr-qc/9905097.

[16] H. Martel and P. R. Shapiro, astro-ph/9903425.

[17] For a discussion and references see A. Vilenkin, Phys. Rev. D 58, 067301 (1998).

[18] A. Vilenkin, Phys. Lett. 117B, 25 (1982); Phys. Rev. D 33, 3560 (1986); J. B. Hartle and S. W. Hawking, ibid. 28, 2960 (1983); A. D. Linde, Lett. Nuovo Cimento Soc. Ital. Fis. 39, 401 (1984).

[19] S. W. Hawking, Phys. Lett. 134B, 403 (1984).

[20] S. Coleman, Nucl. Phys. B310, 643 (1988).

[21] A. Vilenkin, Phys. Rev. D 27, 2848 (1983).

[22] A. D. Linde, Phys. Lett. B 175, 395 (1986).

[23] J. Garriga and A. Vilenkin, Phys. Rev. D 57, 2230 (1998).

[24] J. Garcia-Bellido and A. D. Linde, Phys. Rev. D 51, 429 (1995).

[25] The function $\gamma\left(\rho_{\Lambda}\right)$ can be found as an eigenvalue of the Fokker-Planck operator, as discussed in Refs. [26,27]. With a fixed-time cutoff, as in Eq. (43), this function depends on the choice of the time variable $t[27,28]$. This gauge dependence is usually regarded as a problem and some attempts have been made to define gauge-independent probabilities in the case of a single eternally inflating universe [29]. It appears, however, that in the approach we adopted here for an ensemble of universes it would be unreasonable to require gaugeindependence. The quantity $\gamma\left(\rho_{\Lambda}\right)$ characterizes the rate of growth of the thermalized volume. Clearly, it depends on the time variable with respect to which the rate is calculated. The most natural choice appears to be the proper time which we have used throughout the paper. It is conceivable, however, that there may be other consistent and reasonable approaches which may give different answers for the probability distribution in the case of disconnected universes. Investigation of this issue is left for further research.

[26] A. A. Starobinsky, in Current Topics in Field Theory, Quantum Gravity and Strings, edited by H. J. de Vega and N. Sanchez, Lecture Notes in Physics Vol. 246 (Springer, Heidelberg, 1986), pp. 107-126.

[27] A. D. Linde, D. A. Linde, and A. Mezhlumian, Phys. Rev. D 49, 1783 (1994).

[28] S. Winitzki and A. Vilenkin, Phys. Rev. D 53, 4298 (1996).

[29] A. Vilenkin, Phys. Rev. D 52, 3365 (1995); Phys. Rev. Lett. 81, 5501 (1998).

[30] P. J. E. Peebles and B. Ratra, Astrophys. J., Lett. Ed. 325, L17 (1988); R. R. Caldwell, R. Dave, and P. J. Steinhardt, Phys. Rev. Lett. 80, 1582 (1998); L. Wang, R. R. Caldwell, J. P. 
Ostriker, and P. J. Steinhardt, astro-ph/9901388.

[31] I. Zlatev, L. Wang, and P. J. Steinhardt, Phys. Rev. Lett. 82, 896 (1999).

[32] T. Chiba, Phys. Rev. D 60, 083508 (1999); L. Amendola, astro-ph/9908023.

[33] L. Wang and P. J. Steinhardt, Astrophys. J. 508, 483 (1998).

[34] P. J. E. Peebles and A. Vilenkin, Phys. Rev. D 59, 063505 (1999). 